\title{
Maturação sexual e parâmetros reprodutivos em touros da raça Nelore criados em sistema extensivo
}

\section{Thiago da Silva Silveira ${ }^{1}$, Jeanne Broch Siqueira ${ }^{2}$, Simone Eliza Facioni Guimarães ${ }^{3}$, Tarcízio Antônio Rêgo de Paula ${ }^{4}$, Tamires Miranda Neto ${ }^{5}$, José Domingos Guimarães ${ }^{6}$}

\author{
1 Médico Veterinário autônomo. \\ 2 Pós-doutoranda no Departamento de Zootecnia da Universidade Federal de Viçosa/MG. \\ ${ }^{3}$ Departamento de Zootecnia da Universidade Federal de Viçosa/MG. \\ ${ }^{4}$ Departamento de Medicina Veterinária da Universidade Federal de Viçosa/MG. \\ ${ }^{5}$ CFM-Ltda, São José do Rio Preto/SP. \\ ${ }^{6}$ Departamento de Medicina Veterinária da Universidade Federal de Viçosa/MG (coordenador do Projeto).
}

RESUMO - Objetivou-se verificar o estágio de maturidade sexual, a ocorrência do formato testicular e a relação entre características reprodutivas de tourinhos da raça Nelore. Foram utilizados 5.903 touros com média de 21 meses de idade submetidos a exame andrológico entre os anos de 1999 e 2003. Na ocasião do exame, foram registradas as seguintes características: perímetro escrotal $(31,99 \pm 2,23 \mathrm{~cm})$, comprimentos testiculares esquerdo $(11,21 \pm 0,98 \mathrm{~cm})$ e direito $(11,26$ $\pm 0,97 \mathrm{~cm})$, larguras testiculares esquerda $(5,92 \pm 0,44 \mathrm{~cm})$ e direita $(5,97 \pm 0,46 \mathrm{~cm})$, formato testicular, variando de 1 a 5 $(1,72 \pm 0,46)$, volume testicular $\left(632,21 \pm 132,72 \mathrm{~cm}^{3}\right)$, motilidade espermática progressiva retilínea $(69,56 \pm 12,31 \%)$, vigor espermático $(2,87 \pm 0,61)$ e morfologia espermática, com defeitos totais $(22,19 \pm 11,13 \%)$ e maiores $(15,86 \pm 10,45 \%)$ dos espermatozoides. As freqüências dos formatos testiculares foram longo (30,80\%), longo-moderado (66,19\%), longo-oval $(2,49 \%)$, oval-esférico $(0,02 \%)$ e esférico $(0,04 \%)$. As correlações entre perímetro escrotal e características reprodutivas foram positivas. O perímetro escrotal é uma ótima característica para seleção de touros jovens da raça Nelore, que, na maioria (78,33\%), apresentam-se sexualmente maturos aos 21 meses de idade.

Palavras-chaves: biometria testicular, formato testicular, maturidade sexual

\section{Sexual maturity and reproductive parameters of Nellore bulls, raised under pasture conditions}

\begin{abstract}
The objective of this study was to verify the stage of sexual maturity, occurrence of the testicular format and the relationship among reproductive characteristics. Data were used from 5903 Nellore bulls averaging 21 months submitted to the soundness evaluation from 1999 to 2003. At the time of the evaluation, the following characteristics were recorded: scrotal circumference at soundness evaluation $(31.99 \pm 2.23 \mathrm{~cm})$, left testicular length $(11.21 \pm 0.98 \mathrm{~cm})$ and right testicular length $(11.26 \pm 0.97 \mathrm{~cm})$, left testicular width $(5.92 \pm 0.44 \mathrm{~cm})$ and right testicular width $(5.97 \pm 0.46 \mathrm{~cm})$, testicular format $(1.72 \pm 0.46)$, testicular volume $\left(632.21 \pm 132.72 \mathrm{~cm}^{3}\right)$, individual motility $(69.56 \pm 12.31 \%)$, vigor $(2.87 \pm 0.61)$, as well as total defects $(22.19 \pm 11.13 \%)$ and major defects $(15.86 \pm 10.45 \%)$ of the spermatozoa. The frequencies of the testicular formats were long (30.80\%), long-moderate (66.19\%), long-oval (2.49\%), oval-spherical $(0.02 \%)$ and spherical $(0.04 \%)$. Correlations between the scrotal circumference and the reproductive traits were positive. Scrotal circumference is a good trait for selection of young Nellore bulls. More than $70 \%$ of the animals studied are sexually mature at 21 months old.
\end{abstract}

Key Words: testicle biometric, testicular format, sexual maturity

\section{Introdução}

A utilização de reprodutores selecionados por exame andrológico tem como finalidade a garantia da qualidade seminal e a melhora na eficiência reprodutiva do rebanho visando aumentar a lucratividade média por animal nascido. Esta prática é frequente em rebanhos-elite, porém ainda incipiente na maioria dos rebanhos comerciais. Em estudos e simulações que consideram aumento na proporção touro:vaca da relação tradicional 1:25 para 1:40 e 1:60, seria possível o descarte de $50 \%$ ou mais dos animais com baixo desempenho reprodutivo. Isso resultaria em reduções nos custos de produção, que poderiam ser reinvestidos na aquisição de touros melhoradores,

Recebido em 26/6/2008 e aprovado em 5/3/2009.

Correspondências devem ser enviadas para: jbsiqueira_@hotmail.com 
acelerando o ganho genético no rebanho (Fonseca et al., 1997; Guimarães, 1999).

O aumento da lucratividade pela diminuição da duração do ciclo produtivo, da permanência e a amortização dos custos de mantença do animal na fazenda dependem essencialmente da precocidade sexual média do rebanho. A identificação de touros sexualmente maturos em idades mais precoces, que transmitam esta característica para sua progênie, acarreta grande impacto econômico no sistema produtivo, já que as características reprodutivas em estudos bioeconômicos apresentam valores genéticoeconômicos maiores e são as mais importantes no processo produtivo (Formigoni, 2002).

A maturidade sexual ocorre normalmente 16 a 20 semanas após a puberdade (Lunstra \& Echternkamp, 1982). Animais de origem indiana são extremamente tardios e atingem a maturidade somente aos 30-36 meses de idade (Fonseca et al., 1975). A maioria dos estudos preconiza a maturidade sexual como a idade em que os animais apresentam-se com no mínimo 50\% de motilidade espermática progressiva e morfologia espermática com no máximo de $10 \%$ de defeitos espermáticos maiores e $20 \%$ de defeitos espermáticos menores (Lunstra \& Echternkamp, 1982; Garcia et al., 1987). Como a puberdade é altamente influenciada por fatores de ambiente, principalmente nutrição e condições climáticas, podem ser encontrados animais tanto precoce como tardios quanto a esta característica (Guimarães, 1999).

Este trabalho foi realizado com o objetivo de verificar o estágio de maturidade sexual e sua relação com características reprodutivas em touros da raça Nelore aos 21 meses de idade.

\section{Material e Métodos}

Foram utilizados 5.903 touros jovens da raça Nelore, nascidos nas estações de parição (1 de agosto a 31 de dezembro) dos anos de 1997 a 2001 na fazenda São Francisco, da Agropecuária CFM Ltda (Magda, São Paulo), avaliados por meio de exames andrológicos, realizados no período de julho a novembro de 1999 a 2003. Os animais foram criados a pasto predominantemente de capim Brachiaria decumbens e colonião (Panicum maximum) e, após os 18 meses de idade foram confinados por aproximadamente 60 dias e alimentados com silagem de milho, sal mineral e água à vontade.

Durante o exame andrológico, foi realizada a mensuração de comprimento e largura testicular, com auxílio de paquímetro, e perímetro escrotal, obtido com auxílio de fita métrica na região mais larga do escroto após leve tracionamento ventro-caudal das gônadas. Para determinação do formato testicular, foram adotados os critérios utilizados por Bailey et al. (1996) e Bailey et al. (1998) a partir da razão entre largura e comprimento testicular médio. Os testículos foram classificados em cinco classes: longo (razão $\leq 0,5$ ), longo-moderado (razão de 0,51 a 0,625), longo-oval (razão de 0,626 a 0,750), oval-esférico (razão de 0,751 a 0,875 ) e esférico (razão $\geq 0,875$ ). Empregou-se o formato testicular para determinar o volume testicular, adotando-se as formas preconizadas por Fields et al. (1979) para testículos em formato cilíndrico (longo, longomoderado e longo-oval). Para testículos com formato esférico (quando as gônadas foram classificadas como ovóide-esférico e esférico), foi utilizada a fórmula preconizada por Bailey et al. (1998).

As amostras de sêmen foram obtidas por eletroejaculação. Logo após a coleta, foi realizado o exame das características físicas do ejaculado. Com auxílio de microscópio ótico num aumento de 200 a 400X avaliou-se o movimento em massa dos espermatozoides (turbilhonamento) utilizando-se escala de 1 a 5 , motilidade espermática progressiva retilínea (0-100\%) e o vigor espermático (0-5) (CBRA, 1998). Para análise morfológica dos espermatozoides, uma amostra de sêmen foi acondicionada e estocada em $1 \mathrm{~mL}$ de formol salina tamponada (Hancock, 1957). Nesta avaliação, foi adotada a metodologia preconizada por Blom (1983), registrando-se os defeitos de cabeça, cauda e acrossoma classificando-as em defeitos espermáticos maiores, menores e totais. Foram analisadas 400 células espermáticas por ejaculado, com auxílio de microscópio de contraste de fase, e aumento de 1000X.

A determinação da idade à maturidade sexual foi realizada de acordo com Garcia et al. (1987), cuja definição baseia-se no fato de os animais apresentarem ejaculados com defeitos maiores inferiores a $15 \%$ e defeitos espermáticos totais inferiores a 30\%. Adicionalmente, a maturidade sexual foi classificada segundo Guimarães (1997), conforme estudo do processo espermatogênico, fisiopatologia da reprodução e características físicas e morfológicas dos espermatozoides em: animais sexualmente maturos, aptos à reprodução, de acordo com os padrões recomendados pelo CBRA (1998); animais sexualmente maturos, com índices de patologias espermáticas sem comprometimento da capacidade fecundante das células espermáticas, classificados como aptos à reprodução em regime de monta natural; animais sexualmente imaturos, temporariamente inaptos à reprodução; animais descartados por apresentarem espermiogênese imperfeita severa; animais descartados por apresentarem alterações morfológica dos órgãos genitais. 
As análises estatísticas (médias, desvios-padrão e coeficientes de variação) de todas as características estudadas foram calculadas por meio do PROC MEANS, utilizando-se o pacote estatístico SAS (1999). Os coeficientes de correlação simples de Pearson, para todas as características estudadas, foram estimados pelo PROC CORR (SAS, 1999), determinando a intensidade da associação entre as características estudadas.

\section{Resultados e Discussão}

Em geral, 78,33\% dos animais apresentaram-se maturos sexualmente (classes 1 e 2) com média de idade de 21 meses (Tabela 1). Assim como no trabalho de Vasconcelos (2001), os animais com espermiogênese imperfeita (classe 4) foram redirecionados para a classe 5 , por representarem animais descartados da reprodução, não interferindo desta forma, na discussão dos resultados.

Animais temporariamente inaptos à reprodução $(18,11 \%)$ e descartados (3,56\%), considerados de baixa fertilidade e inférteis respectivamente, somam juntos média de 21,67\%. Vale Filho et al. (1986) encontraram médias de $42,00 \%$ e $40,50 \%$ para baixa fertilidade e infertilidade, respectivamente, em touros Bos taurus indicus mantidos em central de inseminação artificial e touros que serviam como reprodutores em rebanhos de criação extensiva. Considerando os animais sexualmente imaturos e descartados como inférteis, Salvador (2001) e Dias et al. (2007) registraram valores de 59,8 e 73,7\%, respectivamente. $\mathrm{O}$ último autor atribui os resultados à baixa condição nutricional a que estes animais foram submetidos, demonstrando assim, a grande heterogeneidade do rebanho Nelore no Brasil, quanto à maturidade sexual. Os valores obtidos neste estudo demonstram o progresso alcançado para precocidade sexual e melhoramento genético desenvolvido no referido rebanho.

De acordo com os resultados verificados nos cinco anos estudados, 2001 e 2002 apresentaram menor porcentagem de animais aptos à reprodução (<65\%), assim como maior número de animais temporariamente inaptos à reprodução (> 20\%). É possível que uma restrição alimentar na fase pré-puberal e puberal dos animais nascidos nos anos 1999 e 2000 tenha causado o aumento no número de animais inaptos nos anos 2001 e 2002.

Durante o exame andrológico, aqueles animais que apresentaram alterações patológicas que inviabilizam seu uso como reprodutor foram descartados da reprodução (classe 5). Patologias como a vesiculite, espermiogênese imperfeita, assimetria testicular, baixo perímetro escrotal, atrofia testicular, aderência peniana, estenose do esfíncter anal, aprumos, calcificação e/ou fibrose epididimária, hidrocele, papiloma no pênis, persistência do frênulo, tumor de próstata, estenose prepucial, aglutinação espermática e lesão de casco foram encontradas. Assim como observado por Vasconcelos (2001), a vesiculite uni ou bilateral, anomalia provavelmente ocasionada pelo comportamento homossexual ou sodomia entre animais jovens em confinamento com grande número de animais, foi a mais frequente no rebanho.

Entre as variáveis estudadas, os comprimentos testiculares esquerdo e direito, formato testicular e vigor não apresentaram interação ano-classe andrológica ( $p>0,05)$, desta forma, foram analisadas em função do ano de coleta (Tabela 2) e classe andrológica (Tabela 3), independentemente.

Os comprimentos testiculares direito e esquerdo se manifestaram de forma semelhante entre as classes andrológicas (Tabela 3) e igualmente entre os anos (Tabela 2). Vasconcelos (2001) verificou valores médios, semelhantes aos deste estudo, para os comprimentos testiculares em animais da raça Nelore de 20 a 22 meses de idade. Os animais sexualmente maturos (classes 1 e 2) apresentaram maiores valores para comprimentos testiculares direito e esquerdo em comparação aos animais inaptos ou descartados $(\mathrm{p}<0,05)$, resultado também verificado por Dias et al. (2007) em touros da raça Nelore aos 2 anos de idade.

Tabela 1 - Número de animais e frequência de classes andrológicas em cada ano em touros da raça Nelore aos 21 meses de idade criados extensivamente

\begin{tabular}{|c|c|c|c|c|c|c|c|}
\hline \multicolumn{2}{|l|}{ Classe andrológica } & 1999 & 2000 & 2001 & 2002 & 2003 & Total \\
\hline \multirow[t]{2}{*}{ Aptos à reprodução } & $\%$ & 75,25 & 70,34 & 63,43 & 55,84 & 69,16 & 66,49 \\
\hline & $\mathrm{N}$ & $(815)$ & (797) & $(843)$ & $(674)$ & (796) & $(3925)$ \\
\hline \multirow[t]{2}{*}{ Aptos à reprodução em monta natural } & $\%$ & 14,96 & 15,00 & 11,81 & 8,95 & 8,86 & 11,84 \\
\hline & $\mathrm{N}$ & $(162)$ & $(170)$ & $(157)$ & $(108)$ & $(102)$ & (699) \\
\hline \multirow[t]{2}{*}{ Temporariamente inaptos à reprodução } & $\%$ & 7,02 & 9,62 & 24,23 & 29,83 & 17,55 & 18,11 \\
\hline & $\mathrm{N}$ & $(76)$ & (109) & (322) & $(360)$ & $(202)$ & $(1069)$ \\
\hline \multirow[t]{2}{*}{ Animais descartados da reprodução } & $\%$ & 2,77 & 5,03 & 0,53 & 5,39 & 4,43 & 3,56 \\
\hline & $\mathrm{N}$ & $(30)$ & $(57)$ & $(7)$ & $(65)$ & $(51)$ & $(210)$ \\
\hline \multirow[t]{2}{*}{ Total } & $\%$ & 18,35 & 19,19 & 22,51 & 20,45 & 19,50 & 100,00 \\
\hline & $\mathrm{N}$ & $(1083)$ & $(1133)$ & $(1329)$ & $(1207)$ & $(1151)$ & $(5903)$ \\
\hline
\end{tabular}


Tabela 2 - Características reprodutivas (biométricas, físicas e morfológicas do ejaculado) em touros da raça Nelore aos 21 meses de idade criados extensivamente entre os anos de 1999 e 2003

\begin{tabular}{|c|c|c|c|c|c|}
\hline Variável & 1999 & 2000 & 2001 & 2002 & 2003 \\
\hline Comprimento testículo esquerdo & $-*$ & $11,42 \pm 0,06 \mathrm{a}$ & $10,09 \pm 0,10 \mathrm{c}$ & $11,55 \pm 0,06 a$ & $11,09 \pm 0,05 b$ \\
\hline Comprimento testículo direito & $-*$ & $11,50 \pm 0,06 a$ & $10,31 \pm 0,10 \mathrm{c}$ & $11,56 \pm 0,06 a$ & $11,19 \pm 0,05 b$ \\
\hline Formato testicular & $-*$ & $1,75 \pm 0,03 b$ & $2,06 \pm 0,05 a$ & $1,56 \pm 0,03 c$ & $1,54 \pm 0,02 c$ \\
\hline Vigor espermático ( 0 a 5) & $2,57 \pm 0,07 a$ & $2,59 \pm 0,05 a$ & $2,79 \pm 0,07 a$ & $2,67 \pm 0,05 a$ & $2,61 \pm 0,04 a$ \\
\hline
\end{tabular}

Letras iguais na mesma linha não diferem $(\mathrm{p}>0,05)$ pelo teste $\mathrm{T}$.

* Em 1999 não foram realizadas as medidas de comprimento e largura testicular.

Tabela 3 - Características reprodutivas (biométricas, físicas e morfológicas do ejaculado) em touros da raça Nelore de quatro classes andrológicas aos 21 meses de idade

\begin{tabular}{|c|c|c|c|c|}
\hline \multirow[t]{2}{*}{ Variável } & \multicolumn{4}{|c|}{ Classe andrológica } \\
\hline & $\begin{array}{l}\text { Aptos à } \\
\text { reprodução }\end{array}$ & $\begin{array}{l}\text { Aptos à reprodução em } \\
\text { regime de monta natural }\end{array}$ & $\begin{array}{l}\text { Temporariamente } \\
\text { inaptos à reprodução }\end{array}$ & $\begin{array}{l}\text { Descartados da } \\
\text { reprodução }\end{array}$ \\
\hline Comprimento do testítulo direito & $11,35 \pm 0,03 a$ & $11,17 \pm 0,05 b$ & $10,96 \pm 0,04 \mathrm{~b}, \mathrm{c}$ & $11,08 \pm 0,12 \mathrm{c}$ \\
\hline Formato testicular & $1,72 \pm 0,01 \mathrm{a}$ & $1,71 \pm 0,02 \mathrm{a}$ & $1,69 \pm 0,02 \mathrm{a}$ & $1,80 \pm 0,06 a$ \\
\hline Vigor espermático ( 0 a 5) & $2,98 \pm 0,02 a$ & $2,82 \pm 0,03 b$ & $2,40 \pm 0,03 c$ & $2,39 \pm 0,09 c$ \\
\hline
\end{tabular}

Letras iguais na mesma linha não diferem $(\mathrm{p}>0,05)$ entre si pelo teste $\mathrm{T}$.

O ano de coleta não influenciou o vigor (Tabela 2). No entanto, houve diferença $(\mathrm{P}<0,05)$ para esta variável entre as classes andrológicas (Tabela 3 ), uma vez que os animais sexualmente maturos (classes $1 \mathrm{e} 2$ ) apresentaram os maiores valores. Esses resultados foram semelhantes aos observados por Vasconcelos (2001) e Dias et al. (2007) em animais de mesma raça e faixa etária. A média de vigor observados por Costa e Silva (1994), em touros da raça Nelore com mais de 5 anos de idade, divididos em duas categorias (excelentes e bons), foram de 3,64 $\pm 0,35$ e $2,64 \pm 0,71$, respectivamente.

Valores superiores para larguras testiculares foram registrados por Vasconcelos (2001) em touros da raça Nelore dos 20 aos 22 meses de idade (Tabela 4). Valores

Tabela 4 - Média e desvio-padrão dos quadrados mínimos das características biométricas de touros jovens da raça Nelore aos 21 meses de idade criados extensivamente nos anos de 1999 a 2003

\begin{tabular}{|c|c|c|c|c|c|}
\hline & \multirow[b]{2}{*}{ Ano } & \multicolumn{4}{|c|}{ Classe andrológica } \\
\hline & & $\begin{array}{l}\text { Aptos à } \\
\text { reprodução }\end{array}$ & $\begin{array}{l}\text { Aptos à reprodução } \\
\text { em regime de monta natural }\end{array}$ & $\begin{array}{l}\text { Temporariamente } \\
\text { inaptos à reprodução }\end{array}$ & $\begin{array}{l}\text { Descartados da } \\
\text { reprodução }\end{array}$ \\
\hline \multirow{5}{*}{$\begin{array}{l}\text { Perímetro } \\
\text { escrotal (cm) }\end{array}$} & 1999 & $31,98 \pm 0,10 \mathrm{aB}$ & $31,56 \pm 0,19 \mathrm{bA}$ & $30,82 \pm 0,27 \mathrm{cA}$ & $31,04 \pm 0,45 \mathrm{bA}$ \\
\hline & 2000 & $32,12 \pm 0,10 \mathrm{aA}, \mathrm{B}$ & $31,83 \pm 0,18 \mathrm{a}, \mathrm{bA}$ & $31,43 \pm 0,22 \mathrm{bA}$ & $29,47 \pm 0,31 \mathrm{cB}$ \\
\hline & 2001 & $32,17 \pm 0,10 \mathrm{aA}, \mathrm{B}$ & $32,02 \pm 0,19 \mathrm{aA}$ & $31,17 \pm 0,14 \mathrm{bA}$ & $33,02 \pm 0,85 \mathrm{aA}$ \\
\hline & 2002 & $32,06 \pm 0,10 \mathrm{aB}$ & $31,73 \pm 0,22 \mathrm{aA}$ & $30,17 \pm 0,13 \mathrm{bB}$ & $28,31 \pm 0,29 \mathrm{cC}$ \\
\hline & 2003 & $32,35 \pm 0,10 \mathrm{aA}$ & $31,78 \pm 0,23 \mathrm{bA}$ & $31,23 \pm 0,17 \mathrm{cA}$ & $31,61 \pm 0,32 b, c A$ \\
\hline \multirow{5}{*}{$\begin{array}{l}\text { Largura do testículo } \\
\text { esquerdo }(\mathrm{cm})\end{array}$} & 1999 & - & - & - & - \\
\hline & 2000 & $6,10 \pm 0,02 \mathrm{aA}$ & $6,01 \pm 0,04 \mathrm{bA}$ & $5,97 \pm 0,04 \mathrm{bA}$ & $5,94 \pm 0,09 a, b A$ \\
\hline & 2001 & $5,96 \pm 0,02 \mathrm{aC}$ & $5,91 \pm 0,04 \mathrm{aB}$ & $5,77 \pm 0,03 \mathrm{bB}$ & $5,94 \pm 0,17 \mathrm{a}, \mathrm{bA}, \mathrm{B}$ \\
\hline & 2002 & $6,02 \pm 0,02 \mathrm{aB}$ & $5,94 \pm 0,04 \mathrm{aA}, \mathrm{B}$ & $5,66 \pm 0,03 \mathrm{bC}$ & $5,69 \pm 0,08 \mathrm{bB}, \mathrm{C}$ \\
\hline & 2003 & $5,69 \pm 0,02 \mathrm{aD}$ & $5,64 \pm 0,05 a, b C$ & $5,48 \pm 0,03 c D$ & $5,53 \pm 0,06 \mathrm{~b}, \mathrm{cC}$ \\
\hline \multirow{5}{*}{$\begin{array}{l}\text { Largura do testículo } \\
\text { direito }(\mathrm{cm})\end{array}$} & 1999 & - & - & - & - \\
\hline & 2000 & $6,14 \pm 0,02 \mathrm{aA}$ & $6,05 \pm 0,04 \mathrm{bA}$ & $6,00 \pm 0,05 \mathrm{bA}$ & $6,13 \pm 0,09 \mathrm{bA}$ \\
\hline & 2001 & $6,01 \pm 0,02 \mathrm{aC}$ & $6,01 \pm 0,04 \mathrm{aA}$ & $5,82 \pm 0,03 \mathrm{bB}$ & $6,34 \pm 0,18 \mathrm{aA}$ \\
\hline & 2002 & $6,07 \pm 0,02 \mathrm{aB}$ & $5,99 \pm 0,05 a A$ & $5,72 \pm 0,03 \mathrm{bC}$ & $5,74 \pm 0,08 \mathrm{bB}$ \\
\hline & 2003 & $5,70 \pm 0,02 \mathrm{aD}$ & $5,65 \pm 0,05 \mathrm{aB}$ & $5,47 \pm 0,03 \mathrm{bD}$ & $5,65 \pm 0,07 \mathrm{aB}$ \\
\hline \multirow{5}{*}{$\begin{array}{l}\text { Volume } \\
\text { testicular }\left(\mathrm{cm}^{3}\right)\end{array}$} & 1999 & - & - & - & - \\
\hline & 2000 & $699,87 \pm 6,28 \mathrm{aA}$ & $671,42 \pm 11,07 \mathrm{bA}$ & $651,81 \pm 13,40 \mathrm{bA}$ & $651,59 \pm 26,24 \mathrm{a}, \mathrm{bA}$ \\
\hline & 2001 & $588,37 \pm 5,85 \mathrm{aB}$ & $571,25 \pm 11,29 \mathrm{aB}$ & $531,14 \pm 8,25 b C$ & $626,28 \pm 50,66 a, b A, B$ \\
\hline & 2002 & $692,01 \pm 6,06 \mathrm{aA}$ & $660,86 \pm 13,32 \mathrm{bA}$ & $591,25 \pm 7,76 \mathrm{cB}$ & $592,00 \pm 23,61 \mathrm{cA}, \mathrm{B}$ \\
\hline & 2003 & $583,04 \pm 6,00 \mathrm{aB}$ & $567,04 \pm 13,73 \mathrm{aB}$ & $523,43 \pm 10,08 \mathrm{bC}$ & $545,97 \pm 19,35 a, b B$ \\
\hline
\end{tabular}

Médias seguidas de letras minúsculas iguais na mesma linha e letras maiúsculas iguais na mesma coluna não diferiram (p>0,05) entre si pelo teste T. 
médios inferiores, no entanto, foram observados por Dias et al. (2007) em touros da raça Nelore aos 24 meses.

Os valores de perímetro escrotal diferiram entre os animais aptos e inaptos à reprodução $(\mathrm{p}<0,05)$ : as maiores médias foram verificadas para as classes 1 e 2 (32,14 $\pm 0,06$ e 31,79 $\pm 0,10$ cm respectivamente) em comparação à classe de animais temporariamente inaptos à reprodução $(30,94 \pm 0,09 \mathrm{~cm})$. Dias et al. (2007) também verificaram diferença significativa $(p<0,05)$ para valores de perímetro escrotal em animais da raça Nelore maturos e imaturos sexualmente com dois anos de idade. Para as médias de perímetro escrotal, resultados inferiores foram observados por Pinto et al. (1989) e Dias et al. (2007), e semelhantes foram observados por Viu et al. (2006) em touros Nelore com 17 a 20 e 21 a 24 meses de idade $(30,02 \pm 3,03$ e 33,19 $\pm 2,84 c m$, respectivamente). Valentin et al. (2002), trabalhando com touros Nelore de 20 meses, também verificaram média inferior $(27,4 \mathrm{~cm})$ à observada neste estudo. Os valores elevados de perímetro escrotal observados nos animais descartados da reprodução podem ser atribuídos ao fato dos animais terem sido descartados por critérios que muitas vezes não estão relacionados às mensurações testiculares.

Vasconcelos (2001) registrou médias de perímetro escrotal semelhantes às observadas neste estudo em dois rebanhos da mesma empresa, demonstrando que, na população estudada, a média de perímetro escrotal continuou elevada, condizendo com a seleção para perímetro escrotal aplicada nos últimos anos no rebanho. Essas observações refletem avanços no melhoramento genético do rebanho, principalmente quando verificados os padrões descritos para animais da raça Nelore, citados por Fonseca et al. (1989). De acordo com esses autores, os quais acreditam que touros considerados excelentes apresentam $32 \mathrm{~cm}$ de perímetro escrotal dos 24 aos 35 meses de idade.
As correlações entre o perímetro escrotal e larguras testiculares direita e esquerda foram altas (ambas de 0,80 ) e maiores que entre o perímetro escrotal e comprimentos testiculares esquerdo e direito $(0,45$ e 0,43 , respectivamente), demonstrando que o perímetro escrotal é influenciado principalmente pelas larguras e mais fracamente pelos comprimentos testiculares (Tabelas 5 e 6). Tendo em vista o fato de que o perímetro escrotal é medido na região de maior diâmetro testicular, espera-se que animais que possuam menor largura testicular apresentem, consequentemente menor perímetro escrotal. Dias et al. (2008) registraram maiores valores de correlação entre perímetro escrotal e larguras testiculares direita e esquerda (ambas de 0,92), comprimento testicular esquerdo $(0,80)$ e comprimento testicular direito $(0,79)$. Hahn et al. (1969) observaram correlação entre perímetro escrotal com largura do testículo direito e largura do testículo esquerdo de 0,89 (ambas) e entre perímetro escrotal com comprimentos testiculares esquerdo e direito de 0,77 e 0,81 , respectivamente, em animais da subespécie Bos taurus taurus.

Os valores de correlação verificados neste estudo entre os comprimentos e as larguras testiculares e entre os comprimentos e as larguras testiculares entre si (Tabela 5) foram semelhantes aos registrados por Vasconcelos (2001) em animais da raça Nelore dos 20 aos 22 meses de idade e menores que as registradas por Dias et al. (2008) em animais da raça Nelore. Hahn et al. (1969) verificaram maiores valores para as correlações entre comprimentos e larguras testiculares $(0,77$ a 0,80$)$, entre comprimentos $(0,92)$ e larguras $(0,95)$ testiculares entre si, no entanto seus valores foram estimados em animais Bos taurus taurus da raça Holstein, esperando-se que os formatos testiculares destes animais sejam em sua maioria ovalesférico e esférico, valores confirmados por Bailey et al. (1998),

Tabela 5 - Correlações simples de Pearson entre biometria testicular e aspectos físicos do ejaculado de touros da raça Nelore aos 21 meses de idade criados extensivamente

\begin{tabular}{|c|c|c|c|c|c|c|c|c|c|c|c|c|}
\hline & PE & CTE & L T E & CTD & L T D & FOT & VOT & VOL & TURB & MOT & VIG & ASP \\
\hline$P E$ & 1,00 & 0,43 & 0,80 & 0,45 & 0,80 & 0,19 & 0,78 & 0,05 & 0,26 & 0,20 & 0,20 & 0,25 \\
\hline CTE & & 1,00 & 0,42 & 0,95 & 0,42 & $-0,51$ & 0,74 & 0,11 & 0,12 & 0,11 & $*$ ns & 0,11 \\
\hline L TE & & & 1,00 & 0,42 & 0,89 & 0,32 & 0,88 & 0,09 & 0,27 & 0,19 & 0,19 & 0,21 \\
\hline CTD & & & & 1,00 & 0,45 & $-0,50$ & 0,75 & 0,12 & 0,13 & 0,11 & $* n s$ & 0,11 \\
\hline L T D & & & & & 1,00 & 0,30 & 0,89 & 0,08 & 0,27 & 0,19 & 0,19 & 0,21 \\
\hline FOT & & & & & & 1,00 & ${ }^{*} \mathrm{~ns}$ & $* n s$ & 0,07 & 0,04 & 0,11 & 0,05 \\
\hline VOT & & & & & & & 1,00 & 0,12 & 0,24 & 0,17 & 0,13 & 0,20 \\
\hline VOL & & & & & & & & 1,00 & $* \mathrm{~ns}$ & $-0,03$ & $-0,05$ & $* \mathrm{~ns}$ \\
\hline TURB & & & & & & & & & 1,00 & 0,60 & 0,61 & 0,60 \\
\hline MOT & & & & & & & & & & 1,00 & 0,68 & 0,41 \\
\hline VIG & & & & & & & & & & & 1,00 & 0,46 \\
\hline ASP & & & & & & & & & & & & 1,00 \\
\hline
\end{tabular}

$\mathrm{PE}$ = perímetro escrotal na ocasião do exame andrológico; $\mathrm{CTE}=$ comprimento do testículo esquerdo; LTE = largura do testículo esquerdo; CTD = comprimento do testículo direito; LTD = largura do testículo direito; FOT = formato testicular; VOT = volume testicular; VOL = volume do ejaculado; TURB= turbilhonamento; MOT = motilidade; VIG = vigor; ASP = aspecto do sêmen.

$*_{\text {ns }}=$ não-significativo $(\mathrm{p}>0,05)$. 
que também observaram maiores correlações em seus estudos.

O volume testicular apresentou diferenças entre os animais imaturos e maturos sexualmente (Tabela 4), assim como registrado por Dias et al. (2007) em touros da raça Nelore aos 2 anos de idade. Vasconcelos (2001) verificou maiores valores de volume testicular em touros da raça Nelore dos 20 aos 22 meses de idade, oriundos do mesmo rebanho. O volume testicular mostrou-se altamente correlacionado, e em sentido favorável, com o perímetro escrotal (Tabela 5), embora tenha sido menor que o valor de 0,93 registrado por Dias et al. (2008), o que era esperado, já que as correlações entre as biometrias testiculares relatadas pelos autores também foram maiores que as verificadas neste estudo.
Considerando as correlações registradas neste estudo entre perímetro escrotal com volume testicular, biometria testicular, aspectos físicos e morfológicos do ejaculado, associadas à facilidade de obtenção da medida, não se justifica a utilização do volume testicular na seleção de touros da raça Nelore, como sugeriram Bailey et al. (1998). Além disso, o volume testicular apresentou baixos valores de correlação com motilidade $(0,17)$ e vigor $(0,13)$, assim como registrado por Fields et al. (1979) em touros de várias raças na faixa etária de 16 a 20 meses e por Dias et al. (2008) em animais da raça Nelore de 2 e 3 anos de idade.

Os valores para motilidade espermática progressiva retilínea, defeitos espermáticos maiores e totais também apresentaram interação entre ano de coleta e classe andrológica (Tabela 7). As médias de motilidade, defeitos

Tabela 6 - Correlações simples de Pearson entre as características de biometria testicular e aspectos físicos do ejaculado com as características de morfologia espermática do ejaculado de touros da raça Nelore aos 21 meses de idade criados extensivamente

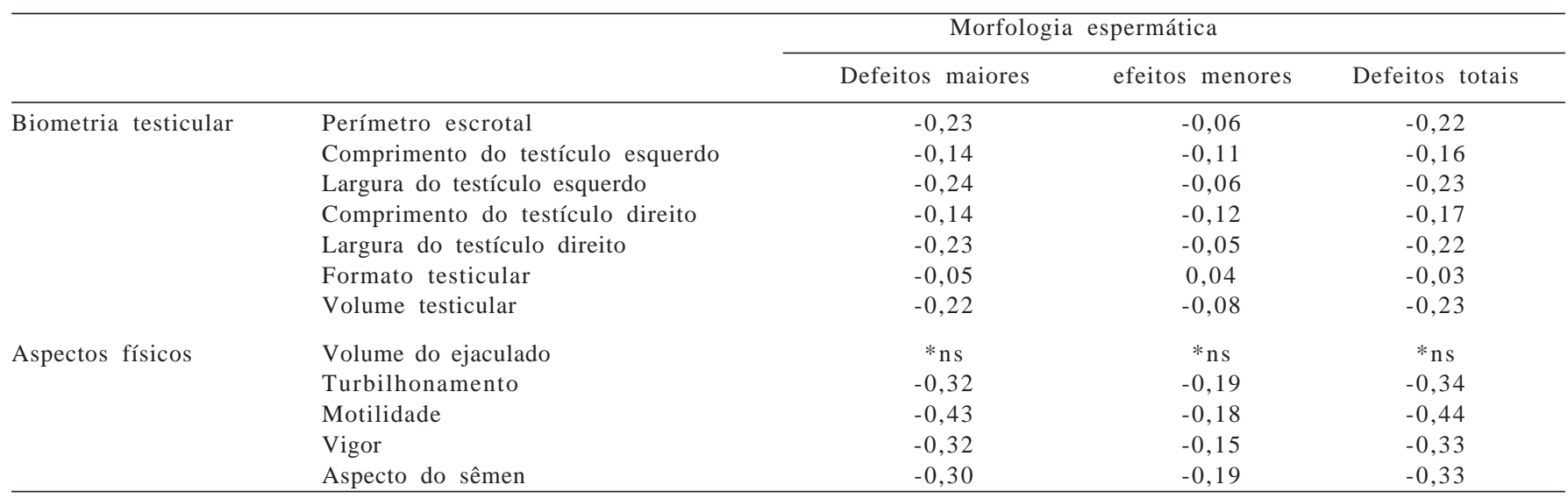

*ns $=$ não-significativo $(\mathrm{p}>0,05)$.

Tabela 7 - Características físicas e morfológicas do ejaculado de touros da raça Nelore aos 21 meses de idade criados extensivamente entre os anos de 1999 e 2003

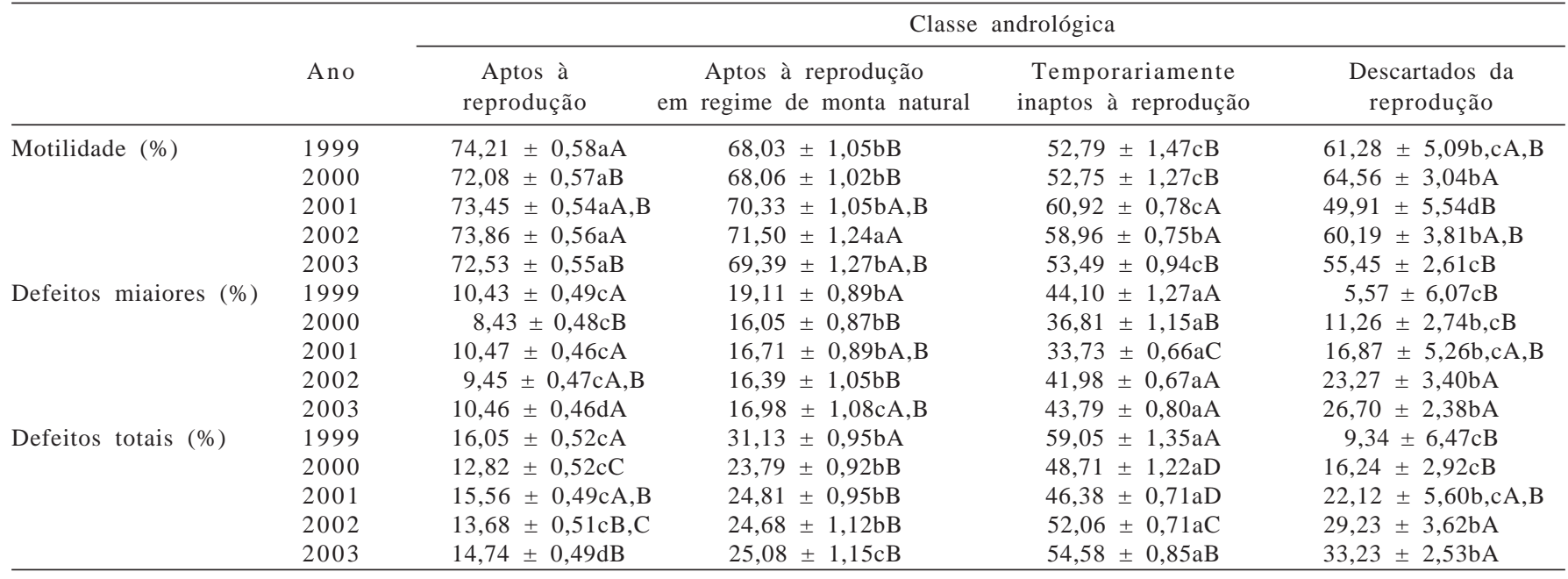

Médias seguidas de letras minúsculas iguais na mesma linha e letras maiúsculas iguais na mesma coluna não diferiram ( $\mathrm{p}<0,05)$ entre si pelo teste $\mathrm{T}$. 
maiores e defeitos totais dos animais maturos sexualmente, classificados neste estudo como aptos à reprodução e aptos à reprodução em regime de monta natural, mantive-ram-se nos padrões para classificação de aptidão dos animais, adotados pelo CBRA (1998) e Chenoweth et al. (1995).

As médias e os desvios-padrão da motilidade para as classes andrológicas foram de 73,23 $\pm 0,31 \%$ (classe 1 ), $69,46 \pm 0,55 \%$ (classe 2), 55,78 $\pm 0,52 \%$ (classe 3) e 58,28 \pm $1,88 \%$ (classe 5 ). Valores de motilidade muito próximos dos observados neste estudo foram relatados por Knights et al. (1984), com média de 63,7 $\pm 12,3 \%$ de espermatozoides vivos para sêmen de touros jovens (12 meses) da raça Angus. Da mesma forma, valores semelhantes foram registrados por Costa e Silva (1994) em touros com mais de 5 anos de idade, considerados excelentes e muito bons em avaliação andrológica. Redução nos valores médios de motilidade entre as classes andrológicas 1 a 3 neste estudo também foi verificada por Vasconcelos (2001) em touros da raça Nelore. Diferença de motilidade entre as classes dentro de cada ano $(\mathrm{p}<0,05)$ também foi observada por Dias et al. (2008) em animais da raça Nelore, maturos e imaturos sexualmente, aos 2 anos de idade.

A relação entre perímetro escrotal com motilidade e vigor $(0,20$ e 0,20$)$ foi próxima à observada por Vasconcelos (2001) em animais da raça Nelore. Silva et al.(2002)verificaram valores maiores de correlação entre perímetro escrotal e motilidade (0,36). Entretanto, quando trabalharam somente com animais de 18 a 24 meses de idade, o valor reduziu para 0,11. Larreal et al. (1988) registraram valores de correlação de 0,61 entre perímetro escrotal e motilidade em animais da raça Nelore de 8 a 30 meses de idade. Salvador (2001) verificou valor negativo $(-0,07)$ para a correlação entre perímetro escrotal e motilidade em animais da raça Nelore dos 20 aos 42 meses de idade. A influência de perímetro escrotal sobre as características físicas do ejaculado, como motilidade, foi variável entre os autores, com correlações positivas e negativas de baixa e média intensidade, o que pode estar relacionado ao fato de esta última variável ser de avaliação subjetiva.

A motilidade apresentou correlação média a alta com vigor (0,68), corroborando estudos de Salvador (2001) e Dias et al. (2008), que relataram correlação de 0,77 e 0,81 entre motilidade e vigor em animais da raça Nelore. Valores menores foram observados por Vasconcelos (2001), que estimou valores de correlação em duas populações de touros da raça Nelore. As características físicas do ejaculado apresentaram correlação entre si, no entanto, se relacionaram negativamente com os defeitos espermáticos, ou seja, com o aumento dos defeitos maiores e totais, a motilidade e o vigor espermático diminuíram.

Para os defeitos maiores, as médias para a classe andrológica 1 e 2 foram (9,85 \pm 0,27\% e 17,05 \pm 0,46\%, respectivamente) semelhantes às encontradas por Dias et al. (2007) e Costa e Silva (1994). Neste estudo, foram verificadas diferenças entre os animais imaturos e maturos sexualmente $(\mathrm{p}<0,05)$, como relatados por Vasconcelos (2001) e Dias et al. (2007) em pesquisa com touros da raça Nelore dos 20 aos 22 meses e dos 2 aos 3 anos de idade, respectivamente.

Os valores médios para defeitos totais foram de $14,57 \pm 0,28 ; 25,90 \pm 0,49 ; 52,16 \pm 0,49$; e 22,03 $\pm 2,02 \%$ para os touros aptos à reprodução; aptos à reprodução em monta natural; os temporariamente inaptos à reprodução e os animais descartados da reprodução. Foram observadas diferenças entre os animais maturos e imaturos sexualmente $(\mathrm{p}<0,05)$, assim como o registrado por Dias et al. (2007) e Vasconcelos (2001). Costa e Silva (1994) verificaram valores $13,43 \pm 4,30$ e 45,93 $\pm 9,00 \%$, respectivamente, de defeitos totais em touros da raça Nelore classificados como excelentes/muito bons e bons andrologicamente. Da mesma forma, Silva et al. (2002) registraram valor médio de $13,4 \pm 13,7 \%$ para defeitos totais em estudo com touros da raça Nelore aos 18 meses de idade.

Os valores de anomalias espermáticas diferiram $(p<0,05)$ entre animais maturos e imaturos sexualmente, uma vez que, de acordo o sistema de classificação para animais jovens proposto por Guimarães (1997), as classes andrológicas são formadas a partir dos valores de anomalias verificados no exame morfológico do sêmen. Os valores baixos de anormalidades espermáticas observados na classe 5 podem ser atribuídos ao fato de os animais terem sido descartados por critérios que muitas vezes não estão relacionados à qualidade seminal.

As correlações entre perímetro escrotal com defeitos maiores, defeitos menores e defeitos totais foram de -0,23; $-0,06$; e -0,22, respectivamente (Tabela 6). De modo semelhante, diversos autores observaram o mesmo comportamento entre esses parâmetros reprodutivos (Salvador, 2001; Vasconcelos, 2001; Silva et al., 2002; Dias et al., 2008).

Todos os valores obtidos para formato testicular, independentemente do ano ou da classe andrológica, foram de 1,5 , ou seja, mais próximos do valor 2 , e indicam que o formato predominante na população estudada foi o longomoderado, com frequência de 66,19\%. Não houve diferença ( $>0,05)$ para formato testicular entre as classes andrológicas (Tabelas 2 e 3). Durante o período avaliado, raramente ocorreram os formatos oval-esférico (um animal) 
Tabela 8 - Número de animais (n) e frequência (\%) do formato testicular em touros da raça Nelore aos 21 meses de idade criados extensivamente

\begin{tabular}{|c|c|c|c|c|c|c|}
\hline \multicolumn{2}{|c|}{ Ano } & \multicolumn{5}{|c|}{ Formato testicular } \\
\hline & & Longo & Longo-moderado & Longo-oval & Oval-esférico & Esférico \\
\hline \multirow[t]{2}{*}{2000} & $\%$ & 32,91 & 64,46 & 2,54 & 0,00 & 0,09 \\
\hline & $\mathrm{N}$ & (363) & (711) & (28) & $(0)$ & (1) \\
\hline \multirow[t]{2}{*}{2001} & $\%$ & 2,11 & 90,06 & 7,76 & 0,08 & 0,00 \\
\hline & $\mathrm{N}$ & $(28)$ & (1196) & (103) & (1) & $(0)$ \\
\hline \multirow[t]{2}{*}{2002} & $\%$ & 43,74 & 55,49 & 0,68 & 0,00 & 0,09 \\
\hline & $\mathrm{N}$ & $(514)$ & $(652)$ & (8) & $(0)$ & $(1)$ \\
\hline \multirow[t]{2}{*}{2003} & $\%$ & 48,70 & 51,22 & 0,09 & 0,00 & 0,00 \\
\hline & $\mathrm{N}$ & $(560)$ & (589) & $(1)$ & $(0)$ & $(0)$ \\
\hline \multirow[t]{2}{*}{ Total } & $\%$ & 30,80 & 66,19 & 2,94 & 0,02 & 0,04 \\
\hline & $\mathrm{N}$ & (1465) & $(3148)$ & $(140)$ & $(1)$ & $(2)$ \\
\hline
\end{tabular}

e esférico (dois animais) (Tabela 8), comprovando que esses formatos testiculares são pouco frequentes em animais da raça Nelore, contrariamente ao observado em animais taurinos da raça Holstein com média de idade de 6,5 anos, que apresentam predominância dos formatos testiculares oval-esférico e esférico (Bailey et al., 1996).

O formato testicular longo-oval (2,94\%) foi verificado nos cinco anos, porém com menor frequência em comparação aos formatos longo e longo-moderado, mas muito expressivo em comparação aos formatos ovalesférico e esférico. Os formatos longo e longo-moderado foram os mais frequentes e, juntos, representaram cerca de 97\% dos formatos testiculares durante os cinco anos de experimento. Resultados semelhantes foram registrados por Vasconcelos (2001), Viu et al. (2006) e Dias et al. (2007) em animais da raça Nelore, o que comprova que os formatos testiculares longo-moderado e longo são predominantes nesta raça.

Houve mudança na frequência do formato testicular entre os anos, com diminuição do formato longo-moderado e aumento do formato longo, praticamente na mesma proporção (aproximadamente 4\%). Entretanto, o formato longo-moderado continuou predominando na população. A predominância dos formatos longo e longo-moderado justifica fisiologicamente a adaptação dos animais da raça Nelore às condições trópico-equatoriais pela teoria proposta por Bailey et al. (1996), na qual os testículos mais alongados apresentam melhor arquitetura vascular, com maior área superficial e melhores distribuições venosas e arteriais, facilitando a perda de calor para o meio. Para esses autores, testículos mais alongados apresentam maior gradiente de temperatura entre os pólos testiculares, resultando em menor temperatura média no microambiente intratesticular, fato comum em animais com ótimos padrões seminais avaliados por termografia via infravermelho (Unanian et al., 2000). Essa conformação testicular confere aos animais Bos taurus indicus maior adaptabilidade aos climas tropicais ou a ambientes com temperaturas médias elevadas (Vale Filho et al., 1986).

As correlações de -0,50 e -0,51 entre formato testicular com comprimento do testículo direito e comprimento do testículo esquerdo, respectivamente, e de 0,30 e 0,32 entre formato testicular com largura do testículo direito e largura do testículo esquerdo, respectivamente, demonstram a importância do comprimento testicular na determinação do formato testicular em animais da raça Nelore na faixa etária estudada. As correlações de formato testicular com volume testicular e perímetro escrotal foram baixas ou nãosignificativas, corroborando os achados de Vasconcelos (2001). Segundo Dias et al. (2007), animais de 2 anos de idade e com testículos longos possuem menor volume testicular e menor perímetro escrotal, indicando, que a esta idade, o perímetro escrotal pode presumir com segurança o tamanho dos testículos. Esses autores também registraram que, em touros aos 3 anos de idade, testículos longos diferiram $(\mathrm{p}<0,05)$ quanto ao perímetro escrotal, porém sem diferença $(\mathrm{p}>0,05)$ quanto ao volume testicular, portanto, nesta idade touros de testículos longos possuem a mesma massa testicular, porém com menor perímetro escrotal.

\section{Conclusões}

Touros Nelore apresentam-se sexualmente maturos entre os 20 e 22 meses de idade. A classificação em cinco classes andrológicas diferencia claramente os animais maturos dos imaturos sexualmente. O perímetro escrotal e o volume testicular estão positivamente correlacionados a todas as características de biometrias testiculares e aspectos físicos e morfológicos do ejaculado, no entanto, considerando a facilidade de obtenção da medida, não se justifica a utilização do volume testicular na seleção de touros da raça Nelore. O formato testicular predominante em animais da raça Nelore aos 21 meses de idade é o longo-moderado. 


\section{Agradecimentos}

À Agro-Pecuária CFM Ltda e ao Sr. Rodney Hobbs (Gerente da fazenda São Francisco - Agro-Pecuária CFM Ltda), pela colaboração e doação das amostras de sêmen e disponibilização dos resultados de campo. Aos pesquisadores que colaboraram no desenvolvimento deste trabalho.

\section{Referências}

BAILEY, T.L.; HUDSON, R.S.; POWE, T.A. et al. Caliper and ultrasonographic measurement of bovine testicles and mathematical formula for determining testicular volume and weight in vivo. Theriogenology, v.49, p.581-594, 1998.

BAILEY, T.L.; MONKE, D.; HUDSON, R.S. et al. Testicular shape and its relationship to sperm production in mature Holstein bulls. Theriogenology, v.46, p.881-887, 1996.

BLOM, E. Pathological conditions in the genital organs and in semen as ground for rejection of breeding bulls for import or export to and from Denmark, 1958-1982. Nordisk Veterinarer Medicin, v.35, p.105-130, 1983.

CHENOWETH, P.J.; HOPKINS F.M.; SPITZER, J.C. et al. Guidelines for using the bull breeding soundness evaluation form. Bovine Short Course. Hastings: American College of Theriogenologists and Society for Theriogernology, 1995. p.111-115.

COLÉGIO BRASILEIRO DE REPRODUÇÃO ANIMAL - CBRA Manual para exame andrológico e avaliação de sêmen animal. 2.ed. Belo Horizonte: CBRA, 1998. 49p.

COSTA e SILVA, E.V. Capacidade reprodutiva de touros Nelore: exame andrológico, teste de comportamento sexual e desafio de fertilidade. 1994. 102f. Dissertação (Mestrado em Ciência Animal) - Universidade Federal de Minas Gerais, Belo Horizonte.

DIAS, J.C.; ANDRADE, V.J.; VALE FILHO, V.R. et al. Biometria testicular e aspectos andrológicos de touros Nelore (Bos taurus indicus), de dois e três anos de idade, criados extensivamente. Veterinária Notícias, v.13, n.2, p.31-37, 2007.

DIAS, J.C.; ANDRADE, V.J.; MARTINS, J.A.M. et al. Correlações genéticas e fenotípicas entre características reprodutivas e produtivas de touros da raça Nelore. Pesquisa Agropecuária Brasileira, v.43, n.1, p.53-59, 2008.

FIELDS, M.J.; BURNS, W.C.; WARNICK, A.C. Age, season and breed effect on testicular volume and semen traits in young beef bulls. Journal of Animal Science, v.48, n.6, p.1299-1304, 1979.

FONSECA, V.O.; CHOW, L.A.; ABREU, J.J. et al. Alguns aspectos físicos e morfológicos de sêmen de touros púberes da raça Nelore. Arquivo da Escola de Veterinária da UFMG, v.27, p.269-75, 1975.

FONSECA, V.O.; FRANCO, C.S.; BERGMANN, J.A.G. et al. Potencial reprodutivo de touros da raça Nelore (Bos taurus indicus) acasalados com elevado número de vacas. Arquivo Brasileiro de Veterinária e Zootecnia, v.49, p.53-62, 1997.

FONSECA, V.O.; CRUDELI, G.O.; SILVA, E.V.C. et al. Classificação andrológica de touros zebus (Bos taurus indicus) com base na biometria testicular e características morfo-físicas do sêmen. Uma nova proposição. Revista Brasileira de Reprodução Animal, supl.1, p.187, 1989.

FORMIGONI, I.B. Estimação de valores econômicos para características componentes de índices de seleção em bovinos de corte. 2002. 80f. Dissertação (Mestrado) - Faculdade de Zootecnia e Engenharia de Alimentos/Universidade de São Paulo, Pirassununga.

GARCIA, J.M.; PINHEIRO, L.E.L.; OKUDA, H.T. Body development and semen physical characteristic of young Guzerá bulls. Arquivo de Veterinária, v.3, n.1, p.47-53, 1987.

GUIMARÃES, J.D. Maximização do uso de touros a campo. In: SIMPÓSIO DE PRODUÇÃO DE GADO DE CORTE, 1., 1999, Viçosa, MG. Anais... Viçosa, MG: Departamento de Zootecnia/ UFV, 1999. p.279-296.

GUIMARÃES, J.D. Avaliação andrológica e estudos quantitativos e qualitativos da espermatogênese de touros mestiços F1 Holandês × Abu e Red Angus × Zebu. 1997. 236f. Tese (Doutorado em Ciência Animal) - Universidade Federal de Minas Gerais, Belo Horizonte.

HAHN, J.; FOOTE, R.H.; SEIDEL JR., G. E. Testicular growth and related sperm output in dairy bulls. Journal of Animal Science, v.29, p.41-47, 1969.

HANCOCK, K.L. The morphology of boar spermatozoa. Journal Reproduction Microscopy Science, v.76, p.84-97, 1957.

KNIGHTS, S.A.; BAKER, R.L.; GIANOLA, D. et al. Estimates of heritabilities and of genetic and phenotypic correlations among growth and reproductive traits in yearling Angus bulls. Journal of Animal Science, v.58, n.4, p.887-893, 1984

LARREAL, H.; TROCONIZ, J.; BELTRAN, J. et al. Scrotal circumference, testicular consistency, body weight and semen traits in Nellore bulls. Journal of Animal Science, v.66, suppl. 1, p.446, 1988.

LUNSTRA, D.D.; ECHTERNKAMP, S.A. Puberty in beef bulls: acrossome morphology and semen quality in bulls of different breeds. Journal of Animal Science, v.55, p.638-648, 1982.

PINTO, P.O.; SILVA, P.R.; ALBUQUERQUE, L.G. et al. Avaliação da biometria testicular e capacidade de monta em bovinos das raças Guzerá e Nelore. Revista Brasileira de Reprodução Animal, v.13, n.3, p.151-156, 1989.

SALVADOR, D.F. Perfis andrológicos, de comportamento sexual e desempenho reprodutivo de touros Nelore desafiados com fêmeas em estro sincronizado. 2001. 53f. Dissertação (Mestrado em Zootecnia) - Universidade Federal de Minas Gerais, Belo Horizonte.

SILVA, O.E.D.F.; UNANIAN, M.M.; CORDEIRO, C.M.T. et al. Relação da circunferência escrotal e parâmetros da qualidade do sêmen em touros da raça Nelore, PO. Revista Brasileira de Zootecnia, v.31, n.3, p.1157-1165, 2002.

STATISTICAL ANALYSIS SYSTEM - SAS. SAS user's guide: Statistics. 8.ed. Cary: SAS Institute, 1999. 956p.

UNANIAN, M.M.; SILVA, O.E.D.F.; McMANUS, C. et al. Características biométricas testiculares para avaliação dos touros zebuínos da raça Nelore. Revista Brasileira de Zootecnia, v.29, n.1, p.136-144, 2000.

VALE FILHO, V.R.; PINHEIRO, L.E.L.; BASRUR, P.K. Reproduction in zebu cattle. In: MORROW, D.A. (Ed.) Current therapy in theriogenology. 2.ed. Philadelphia: W.B. Saunders Company, 1986. p.437-442.

VALENTIM, R.; ARRUDA, R.P.; BARNABE, R.C. et al. Biometria testicular de touros nelore (Bos taurus indicus) e touros cruzados Nelore-europeu (Bos taurus indicus x Bos taurus taurus) aos 20 e 24 meses de idade. Brazilian Journal of Veterinary Research and Animal Science, v.39, p.113-120, 2002.

VASCONCELOS, C.O.P. Estádio de maturidade sexual em touros da raça Nelore, dos 20 aos 22 meses de idade. 2001. 59f. Dissertação (Mestrado em Medicina Veterinária) Universidade Federal de Viçosa, Viçosa, MG.

VIU, M.A.O.; MAGNABOSCO, C.U.; FERRAZ, H.T. Desenvolvimento ponderal, biometria testicular e qualidade seminal de touros Nelore (Bos taurus indicus) criados extensivamente na região centro-oeste do Brasil. Archives of Veterinary Science, v.11, n.3, p.53-57, 2006. 